(c) American Dairy Science Association, 2005.

\title{
Distribution and Carbohydrate Structures of High Molecular Weight Glycoproteins, MUC1 and MUCX, in Bovine Milk*
}

\author{
C. Liu, ${ }^{1,} †$ A. K. Erickson, ${ }^{2}$ and D. R. Henning ${ }^{1}$ \\ ${ }^{1}$ Dairy Science Department, and \\ ${ }^{2}$ Veterinary Science Department, South Dakota State University, Brookings 57007
}

\begin{abstract}
High molecular weight glycoproteins, MUC1 and MUCX, originating from bovine milk, were compared with regard to their distribution in milk fat and skim milk fractions and for presence of carbohydrate structures. Polymorphic MUC1, which migrated into 6\% resolving SDS-PAGE gels, was found in both milk fat globule membrane and skim milk phases of bovine milk. In contrast, MUCX, appearing as a non-polymorphic single band in $3 \%$ polyacrylamide stacking gels, was present only in the skim milk fraction. Peptide-N-glycosidase $\mathrm{F}$ digestion studies indicated that MUC1 and MUCX possessed N-glycans with MUC1 containing more N-glycans than MUCX. Exoglycosidase digestion studies revealed the existence of abundant terminal sialic acid residues in both MUC1 and MUCX. Lectinbinding studies showed that MUCX likely possessed more complex carbohydrate structures than MUC1. The complex carbohydrate structures carried by both MUC1 and MUCX suggest that they may have potential to bind a wide spectrum of pathogenic microorganisms. If that proves to be the case in vivo, such structures could have a role in preventing or reducing some infectious diseases.
\end{abstract}

(Key words: bovine milk, MUC1, MUCX, carbohydrate structures)

Abbreviation key: Gal = galactose, $\mathbf{M F G M}=$ milk fat globule membrane, PAS = periodic acid-Schiff's, PNGase F = Peptide-N-glycosidase F, PBS-TW = PBS containing $0.05 \%$ Tween $20, \mathbf{P V D F}=$ polyvinylidene difluoride, WGA = wheat germ agglutinin.

Received May 9, 2005.

Accepted August 19, 2005.

Corresponding author: David R. Henning, E-mail: David.Henning @sdstate.edu.

*Published with the approval of the Director of South Dakota Agricultural Experiment Station as Publication number 3494 of the Journal Series.

$\dagger$ Current address: Department of Animal Sciences, Ohio Agricultural Research and Development Center, The Ohio State University, Wooster 44691.

\section{INTRODUCTION}

Mucins are a diverse family of heavily glycosylated proteins. Three different mucins, MUC1, MUCX, and MUC15, have been described in the milks of a number of species including bovine (Patton et al., 1995; Pallesen et al., 2002). All of these mucins originate from the apical plasma membrane of lactating cells and are components of milk fat globule membranes (MFGM). As one of periodic acid-Schiff's (PAS) reagent-stainable glycoproteins in MFGM (Mather, 2000), bovine milk MUC1, also known as PAS I, has been described as polymorphic bands on SDS-PAGE gels (Patton and Patton, 1990; Patton and Muller, 1992; Huott et al., 1995). This is mainly due to the existence of variable numbers of tandem repeats domain in its core protein (Pallesen et al., 2001). Bovine MUC1 carries both O- and N-glycans that are composed of $\mathrm{N}$-acetylgalactosamine, $\mathrm{N}$ acetylglucosamine, galactose (Gal), mannose, sialic acid, and fucose (Snow et al., 1977; Pallesen et al., 2001). Bovine MUCX has been observed as a single nonpolymorphic band on SDS-PAGE gels (unpublished data; Patton, 2001). Limited information is available for bovine MUCX with regard to the structure of either its core protein or its carbohydrate moiety. Another bovine MFGM protein, PAS III, has also been identified as a mucin-type glycoprotein and named as MUC15 in a recent study (Pallesen et al., 2002). In the current study, we report the results comparing the distribution and carbohydrate structures of MUC1 and MUCX from bovine milk.

\section{MATERIALS AND METHODS}

\section{Materials}

Polyvinylidene difluoride (PVDF) membrane Immobilon-P, PVDF membrane Immobilon-P, and $10 \mathrm{~K}$ Minicon Concentrator were purchased from Millipore (Bedford, MA). Vibrio cholerae neuraminidase and $\beta$ galactosidase were obtained from Boehringer Mannheim Corp. (Indianapolis, IN). Horseradish peroxidasestreptavidin conjugate was purchased from Pierce Biotechnology (Rockford, IL). Biotinylated lectins and a 3,3'-diaminobenzidine substrate kit were obtained from 
Vector Laboratories (Burlingame, CA). Schiff's reagent, alcian blue, glutaraldehyde, bovine serum albumin, Peptide-N-glycosidase F (PNGase F), and Ponceau S were obtained from Sigma-Aldrich Corp. (St. Louis, MO). The other chemicals were purchased from Fisher Chemicals (Fair Lawn, NJ).

\section{Methods}

Milk sample preparation. Individual bovine (Holstein) milk samples were obtained from the South Dakota State University Dairy Research Unit. All milk samples were taken at the beginning of the second milking of the day. Milk fat globules were isolated according to the method described by Patton and Huston (1986). Sucrose was added to a $30-\mathrm{mL}$ fresh whole milk sample to yield a final sucrose concentration of $5 \mathrm{~g} / 100 \mathrm{~mL}$. The treated milk was delivered under a layer of distilled water $(15 \mathrm{~mL})$ in a $50-\mathrm{mL}$ plastic centrifuge tube. The tube was centrifuged $(2000 \times g, 20 \mathrm{~min}$, room temperature). The top layer, mainly consisting of washed milk fat globules, was transferred into a new tube. The original tube was then centrifuged $(3000 \times g, 2 \mathrm{~h}$, room temperature) and frozen at $-20^{\circ} \mathrm{C}$. The water layer of the frozen tube was cut off to obtain the skim milk. Skim milk whey was obtained by adjusting the skim milk to $\mathrm{pH} 4.6$ with addition of acetic acid followed by removal of the casein precipitate by centrifugation $(3000 \times g, 10 \mathrm{~min}$, room temperature). Milk fat globule membranes were prepared by the addition of $0.2 \%$ Triton X-100 to the washed milk fat globules followed by centrifugation $\left(100,000 \times g, 2 \mathrm{~h}, 4^{\circ} \mathrm{C}\right.$; Patton, 1982).

SDS-PAGE and Western blotting. Protein concentrations were estimated by the procedure described by Peterson (1983). Samples were solubilized in an equal volume of $2 \times$ sample buffer [ $125 \mathrm{~m} M$ Tris- $\mathrm{HCl}, 4 \%$ SDS, $20 \%$ glycerol, $10 \%$ 2-mercaptoethanol, $0.004 \%$ bromophenol blue] and separated by SDS-PAGE gels (Laemmli, 1970). Gels were either stained directly or electrotransferred to PVDF membrane Immobilon-P according to the procedure described by Towbin et al. (1979).

Protein staining on gels. The PAS reagent staining procedure was modified from the method described by Neville and Glossmann (1974). Gels were sequentially soaked in fixing solution (40\% methanol, $7 \%$ acetic acid), oxidation solution (1\% periodic acid, $7 \%$ acetic acid), wash solution (7\% acetic acid), and Schiff's reagent. Incubation times for each solution were $30 \mathrm{~min}$. The stained gels were finally washed in a large volume $(>1 \mathrm{~L})$ of water until the background was clear.

Silver staining was performed as described by Morrissey et al. (1981) with a final-step modification. At first, proteins were fixed in the gel by incubating the gel in $40 \%$ methanol and $7 \%$ acetic acid for $30 \mathrm{~min}$ and in $10 \%$ glutaraldehyde for $10 \mathrm{~min}$. Gels were rinsed in a large volume $(>1 \mathrm{~L}$ ) of water for $>2 \mathrm{~h}$ and then soaked in $5 \mu \mathrm{g} / \mathrm{mL}$ dithiothreitol for $30 \mathrm{~min}$. Gels were incubated in $0.1 \%$ silver nitrate for $30 \mathrm{~min}$. After being rinsed with water for $2 \mathrm{~min}$, gels were then incubated in $3 \%$ sodium carbonate containing $0.019 \%$ formaldehyde until the desired color developed. Finally, the gel was washed in 5\% acetic acid to stop the reaction.

Protein staining on polyvinylidene difluoride membranes. Alcian blue staining of PVDF membrane was performed as described previously (Akiba et al., 2000) with some minor modifications. The membrane was blocked with $2 \%$ BSA for 10 min followed by incubation in $0.5 \%$ alcian blue, $3 \%$ acetic acid ( $\mathrm{pH} 2.5$ for 2 min) and then a water rinse to clear the background.

For lectin binding, the membranes were washed with phosphate-buffered saline $\left(15 \mathrm{~m} M \mathrm{KH}_{2} \mathrm{PO}_{4}, 8 \mathrm{~m} M\right.$ $\mathrm{Na}_{2} \mathrm{HPO}_{4}, 137 \mathrm{mM} \mathrm{NaCl}$, and $2.6 \mathrm{mM} \mathrm{KCl}, \mathrm{pH}$ 7.4) containing $0.05 \%$ Tween 20 (PBS-TW) 3 times for 10 min each and incubated with biotinylated lectins (10 $\mu \mathrm{g} / \mathrm{mL}$ ) in PBS-TW for $30 \mathrm{~min}$. The membranes were washed with PBS-TW 3 times for 10 min and incubated with horseradish peroxidase-streptavidin conjugate (10 $\mu \mathrm{g} / \mathrm{mL}$ ) in PBS-TW for $30 \mathrm{~min}$. The membranes were washed 3 times for 10 min each with PBS-TW, and the bound horseradish peroxidase-streptavidin was detected using 3,3'-diaminobenzidine substrate kit for peroxidase according to the manufacturer's protocol.

Peptide-N-glycosidase $\boldsymbol{F}$ digestion. Skim milk whey samples were first denatured by incubating in $0.5 \%$ SDS and $1 \%$ 2-mercaptoethanol at $100^{\circ} \mathrm{C}$ for 10 min. Then, samples were digested in reaction buffer [50 $\mathrm{m} M$ sodium phosphate (pH 7.5); 1\% NP-40] supplemented with PNGase F (5 U/mg of sample protein) at $37^{\circ} \mathrm{C}$ for $16 \mathrm{~h}$. Buffer exchanges were accomplished using $10 \mathrm{~K}$ Minicon Concentrator according to manufacturer's guide.

Exoglycosidase treatment. Skim milk whey samples were digested with Vibrio cholerae neuraminidase $(0.15 \mathrm{mU} / \mathrm{mL}$ of whey) in digestion buffer $(50 \mathrm{~m} M$ sodium acetate, $150 \mathrm{~m} M \mathrm{NaCl}, 9 \mathrm{mM} \mathrm{CaCl}_{2}$; $\mathrm{pH}$ 5.5) at $37^{\circ} \mathrm{C}$ for $16 \mathrm{~h}$ or with $\beta$-galactosidase $(0.05 \mathrm{mU} / \mathrm{mL}$ of whey) in digestion buffer [50 $\mathrm{m} M$ sodium phosphate, 1 $\mathrm{m} M$ dithiothreitol, and $100 \mathrm{mM} \mathrm{NaCl} ; \mathrm{pH} 7.3$ ] at $37^{\circ} \mathrm{C}$ for $16 \mathrm{~h}$. Buffer exchanges were accomplished with 10 $\mathrm{K}$ Minicon Concentrator according to the manufacturer's guide.

Amino acid compositional analysis. Skim milk whey samples (50 $\mu \mathrm{g}$ per lane) were electrophoresed on SDS-PAGE (3 and 6\% polyacrylamide for stacking and resolving gels, respectively) and transferred to PVDF membrane Immobilon-P as just described. The membrane was stained with Ponceau S $0.2 \%$ in $1 \%$ acetic acid) to visualize lanes. To determine the location of 
mucin bands, one lane was cut off and stained with alcian blue as described before. This lane was used as a reference lane for cutting the mucin bands from the other lanes. The cut membrane pieces containing the mucin bands were rinsed with water and dried. The dried membrane slices were sent to Commonwealth Biotechnologies (Richmond, VA) for AA compositional analysis. Briefly, the PVDF membranes were hydrolyzed in gas phase $6 \mathrm{~N} \mathrm{HCl}$ for $90 \mathrm{~min}$ at $150^{\circ} \mathrm{C}$. Following hydrolysis, the samples were dried, and the AA were extracted in $100 \mu \mathrm{L}$ of $40 \%$ methanol, $0.1 \mathrm{~N}$ $\mathrm{HCl}$ in HPLC water for $16 \mathrm{~h}$. The extracted AA were subjected to analysis using HPLC.

\section{RESULTS AND DISCUSSION}

Figure 1A shows that both MUC1 and MUCX were identified in bovine milk from one cow using silver, PAS, and alcian blue staining methods. Figure $1 \mathrm{~B}$ shows the typical appearance of milk MUC1 and MUCX from 3 different cows (12 cows were examined). MUC1 was detected as polymorphic bands (1 or 2), whereas MUCX, which remained in the stacking gel, appeared as a nonpolymorphic single band in the milks from different cows. Because staining of MUC1 by Morrissey's (1981) silver staining method develops faster than staining of most other proteins, this procedure has been used to selectively stain milk MUC1 on SDS-PAGE gels (Patton and Patton, 1990; Patton and Muller, 1992). The reason that this silver staining procedure stains mucins first is unclear. However, it may be related to the carbohydrate content or to the presence of specific types of carbohydrates such as sialic acid. In the current study, glycoproteins were always stained before non-glycosylated proteins by this silver staining method. In addition, PNGase $\mathrm{F}$ and neuraminidase treatments of mucins decreased the intensity of mucin bands and increased the time needed for developing mucin bands (Figures 2, B and C). The current study also found that MUCX bands were stained more rapidly than MUC1 bands, implying that MUCX may have more carbohydrate than MUC1. Both PAS, which stains carbohydrates (McManus, 1946), and alcian blue, which stains sulphate and carboxyl groups (Pearse, 1980), stained bovine milk MUC1 and MUCX, suggesting that these mucins carry a substantial number of carbohydrate and acidic groups. Earlier reports indicate that the carbohydrate content is around 50\% for human (Shimizu and Yamauchi, 1982) and bovine (Snow et al., 1977) milk MUC1. Milk MUC1 is markedly acidic, mainly because of the abundant sialic acid content, which accounts to about $11 \%$ of total carbohydrate in human milk MUC1 (Shimizu and Yamauchi, 1982), 30\% in bovine milk
$\mathbf{A}$

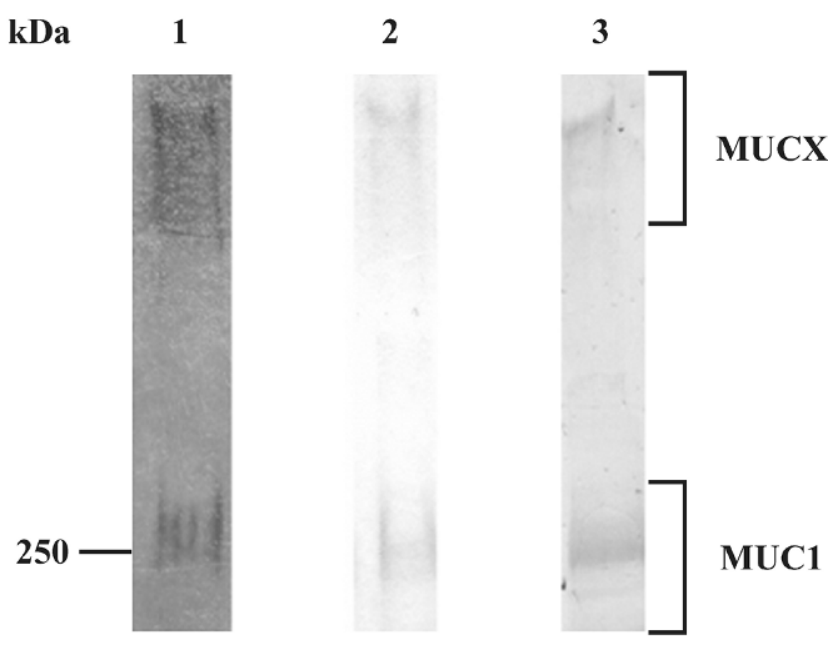

B

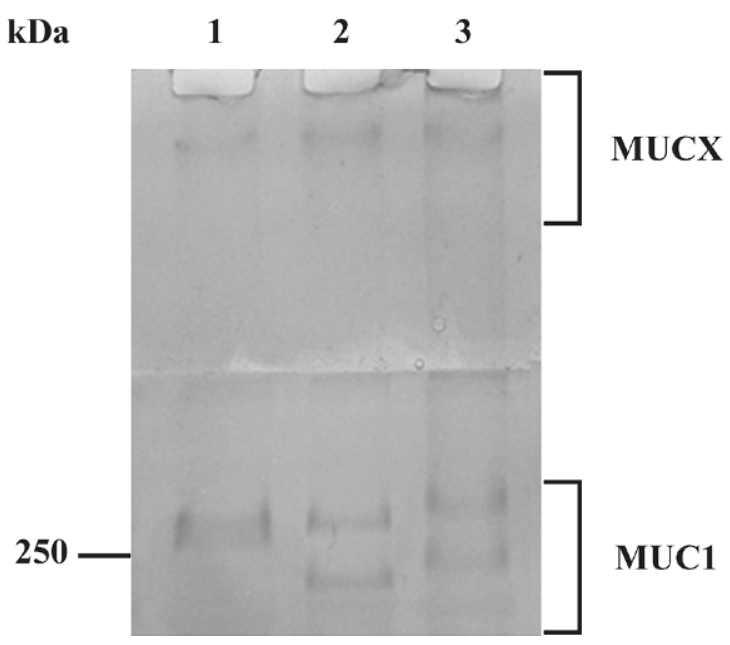

Figure 1. Detection and appearance of MUC1 and MUCX in bovine milk. A) Skim milk whey samples from one cow were stained by silver (lane 1) and periodic acid-Schiff's (PAS; lane 2) or transferred to polyvinylidence difluoride membranes and stained by alcian blue (lane 3). B) Skim milk whey samples from 3 different cows (lanes 1 to 3 ) were stained by silver. All samples (20 $\mu \mathrm{g}$ per lane) were separated by SDS-PAGE (3 and 6\% polyacrylamide for stacking and resolving gels, respectively).

MUC1 (Snow et al., 1977), and 60\% in guinea pig milk MUC1 (Johnson et al., 1988).

The distribution of MUC1 and MUCX was investigated in the various fractions of milk from one cow. MUC1 was detected in all milk fractions (whole milk, skim milk, skim milk whey, and MFGM), whereas MUCX was not detected in MFGM (Figure 2A). The lack of MUCX in bovine MFGM preparations implies 
A

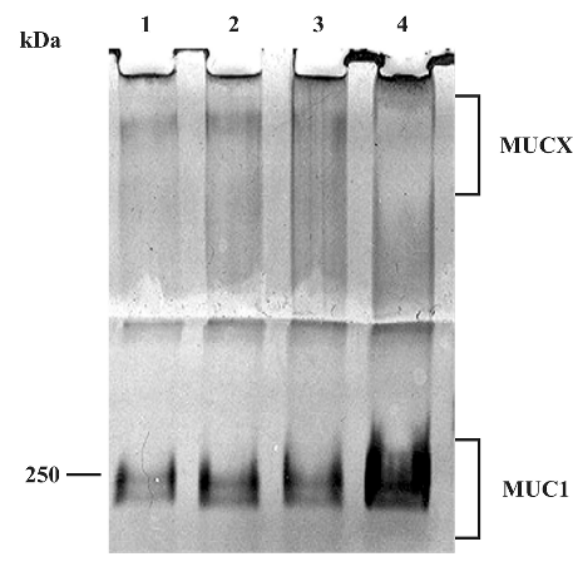

B

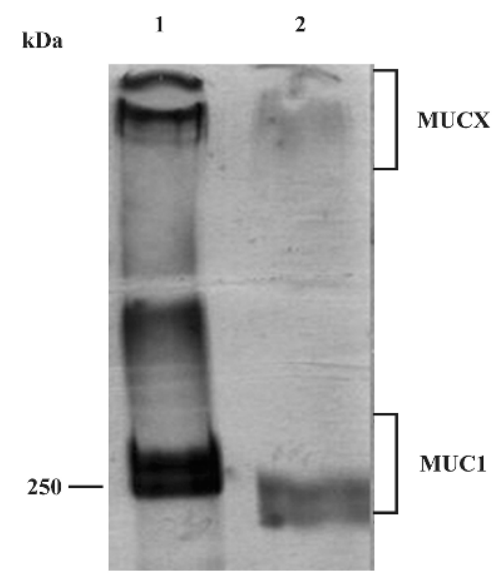

C

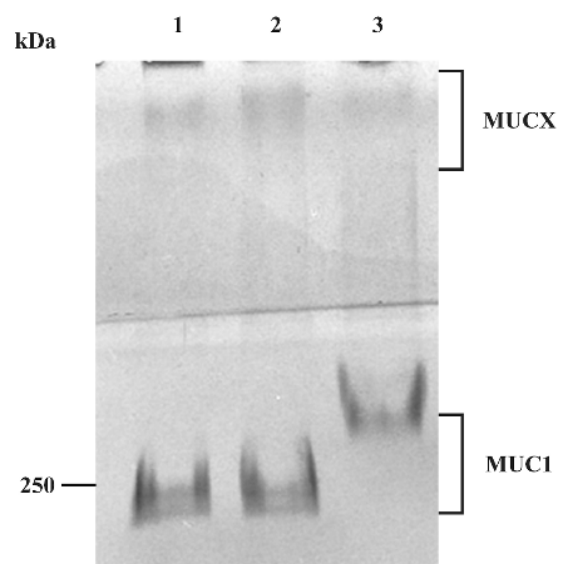

Figure 2. Distribution and characterization of bovine milk MUC1 and MUCX. A) Bovine whole milk (lane 1), skim milk (lane 2), skim milk whey (lane 3), and milk fat globule membrane (lane 4). B) Bovine skim milk whey samples were treated in the absence (lane 1) or presence (lane 2) of Peptide-N-glycosidase F. C) Bovine skim milk whey samples were subjected to treatment with no addition (lanes 1 ), $\beta$-galactosidase (lanes 2 ), and neuraminidase (lane 3 ). All samples (20 $\mu \mathrm{g}$ per lane) were separated by SDS-PAGE (3 and 6\% polyacrylamide for stacking and resolving gels, respectively) and stained by silver. that MUCX may be more loosely associated with MFGM and easier to dislodge from MFGM than MUC1. A possible looser association of MUCX with MFGM may also explain why an earlier study failed to detect MUCX in bovine MFGM (Patton et al., 1989). In the current study, both MUC1 and MUCX were observed to be present in the skim milk phase. Previously, MUC1 has been also reported to exist in skim milk of several species including bovine (Shimizu et al., 1986; Patton and Patton, 1990; Campana et al., 1992). Molecular evidence indicates that human MUC1 protein is cleaved into a heterodimer before it is presented on the cell surface, and the resultant 2 subunits are associated together by non-covalent interaction (Ligtenberg et al., 1992). Not surprisingly, such noncovalent interaction may be easily disrupted during MFGM preparation. Spliced MUC1 mRNA variants lacking membrane anchors, along with their protein products, have been described in humans (Baruch et al., 1999). Therefore, the potential sources of skim milk MUC1 may include broken MFGM fragments or MFGM associated with very small milk fat globules, disrupted MUC1 dimers, and spliced MUC1 variants lacking membrane anchors. Similar to MUC1, rat sialomucin complex (rat Muc4; a putative candidate of milk MUCX) is also present as a heterodimer on the cell surface (Rossi et al., 1996), and human MUC4 has alternatively spliced variants with truncated membrane anchors (Escande et al., 2002). Thus, the potential sources of skim milk MUCX are probably similar to those of MUC1. Interestingly, a recent study indicates that rat sialomucin complex is released from the cell surface not by the speculated shedding mechanism but by an intracellular proteolytic cleavage event that is different from the cleavage to produce the 2 subunits (Komatsu et al., 2002).

Endoglycosidase PNGase F, which specifically cleaves $\mathrm{N}$-glycans from the glycoproteins they are attached to, was used to examine the presence of $\mathrm{N}$ glycans on bovine milk mucins. Treatment of bovine skim milk whey samples with PNGase F led to a large shift in the mobility of MUC1 (Figure 2B), indicating the presence of substantial N-glycans on MUC1. This observation is consistent with the results from the previous study which also observed increased mobility of bovine MUC1 after PNGase F treatment (Pallesen et al., 2001). In contrast, the mobility of MUCX was not greatly affected, although the band density was decreased (Figure 2B), implying that at least a small amount of N-glycans is present on MUCX. From the deduced AA sequence data of bovine MUC1, 5 potential $\mathrm{N}$-glycosylation sites (Asn-X-Ser/Thr, where $\mathrm{X}$ is any AA except Pro) can be identified between the variable number of tandem repeats (VNTR) region and transmembrane domain (Pallesen et al., 2001). The PNGase 
Table 1. Lectin binding to bovine milk MUC1 and MUCX.

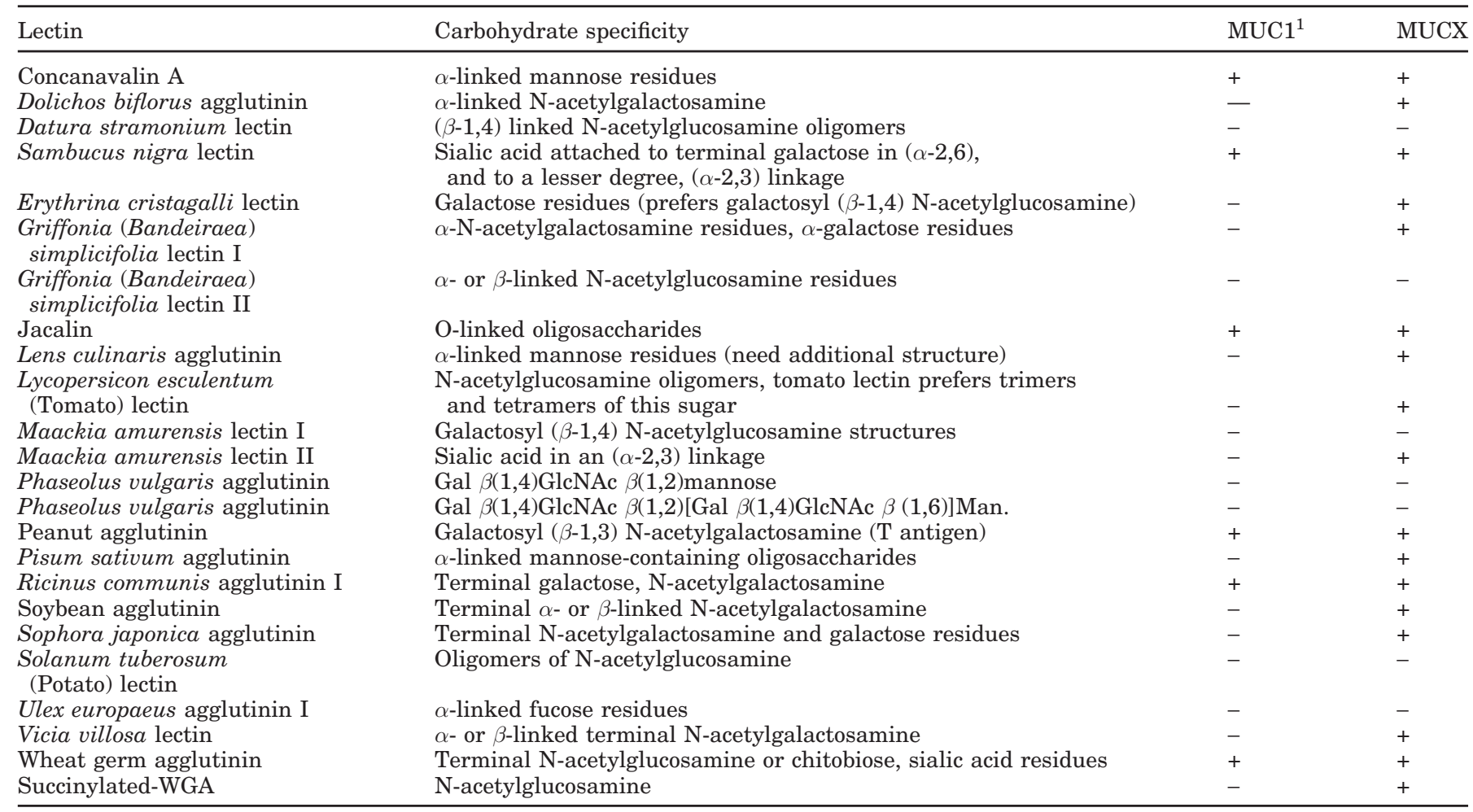

${ }^{1}+=$ positive lectin staining; $-=$ no detectable lectin binding.

F digestion results in the current study confirmed that at least some of these 5 potential N-glycosylation sites are glycosylated in bovine milk MUC1.

To determine the type of sugars found in the terminal position of milk mucin glycans, skim milk whey samples were treated with exoglycosidases (Figure 2C). Treatment of skim milk whey samples with neuraminidase reduced the mobility of both MUC1 and MUCX on SDSPAGE gels. Treatment of skim milk whey samples with $\beta$-galactosidase slightly increased the mobility of MUC1 and MUCX. Compared with MUC1, MUCX showed smaller mobility changes as the result of both neuraminidase and $\beta$-galactosidase treatments. The obvious decrease in mobility of milk mucins after neuraminidase treatment is a reflection of the existence of abundant terminal sialic acid residues on milk mucins. The carboxyl groups on sialic acid make this type of sugar highly negatively charged. In SDS-PAGE, usually the native charge of a protein can be effectively masked by SDS. However, in the case of milk mucins, the large amount of sialic acid residues may significantly contribute to the overall negative charge of the SDS-glycoprotein complex, such that the loss of negative charge caused by the removal of sialic acid residues has a greater effect on the mobility of mucins in SDS-PAGE gels than the loss of mass. Guinea pig MUC1 (Johnson et al., 1988) and bovine MUC1 (Huott et al., 1995) show similar mobility changes on SDS-PAGE gels after neuraminidase treatment. In the current study, a slight increase in mobility occurred for both MUC1 and MUCX after $\beta$-galactosidase treatment, suggesting the presence of some terminal Gal in bovine milk mucins. Previously, a substantial amount of Gal has also been reported in human (Shimizu and Yamauchi, 1982), bovine (Snow et al., 1977), and guinea pig (Johnson et al., 1988) milk MUC1.

Twenty-four lectins were used to further characterize the carbohydrate structures of the glycans present on milk mucins (Table 1). Jacalin, a lectin that is specific for O-glycosylation core structure with preference to $\operatorname{Gal} \beta(1,3) \mathrm{N}$-acetylgalactosamine sequence ( $\mathrm{T}$ antigen), strongly bound both MUC1 and MUCX. This result fits the fact that mucins are highly O-glycosylated proteins. Wheat germ agglutinin (WGA) strongly bound both mucins as well. Wheat germ agglutinin has been reported to bind human MUCX, human MUC1, and bovine MUC1 (Shimizu and Yamauchi, 1982; Shimizu et al., 1986; Patton et al., 1989). The binding activity of WGA seemed to be associated with sialic acid residues of milk mucins because the current study found the WGA binding activity for mucins was greatly reduced or even disappeared after milk samples were treated with neur- 
Table 2. Amino acid compositions of bovine milk MUC1 and MUCX.

\begin{tabular}{lrr}
\hline $\mathrm{AA}^{1}$ & MUC1 & MUCX \\
\cline { 2 - 3 } & & \\
\cline { 2 - 3 } As(X) & 8.6 & 7.5 \\
Gl(X) & 7.0 & 7.6 \\
Ser & 14.3 & 13.1 \\
His & 1.9 & 1.3 \\
Gly & 11.5 & 11.6 \\
Thr & 8.9 & 13.9 \\
Ala & 10.0 & 6.0 \\
Arg & 3.6 & 2.6 \\
Tyr & 2.0 & 1.2 \\
Val & 4.9 & 6.3 \\
Met & 1.4 & 2.1 \\
Phe & 3.1 & 2.9 \\
Ile & 3.9 & 3.7 \\
Leu & 7.0 & 7.3 \\
Lys & 3.4 & 2.5 \\
Pro & 7.9 & 10.4 \\
\hline
\end{tabular}

${ }^{1}$ Cys and Trp were not determined.

aminidase (data not shown). Ricinus communis agglutinin I, which is specific for terminal D-Gal residues, bound both mucins, but its binding was weaker than Jacalin and WGA binding. This is another indication of the existence of terminal Gal residues and is consistent with the result of the $\beta$-galactosidase digestion study. Overall, MUCX is bound by more lectins than MUC1, with some lectins (e.g., Dolichos biflorus agglutinin, Lycopersicon esculentum) binding to MUCX but not to MUC1, implying that MUCX carries more complicated and variable carbohydrate structures.

The results from AA compositional analysis indicate that both bovine milk MUC1 and MUCX have high Ser, Thr, and Pro contents (Table 2), which is a typical feature for mucin proteins (Patton et al., 1995).

\section{CONCLUSIONS}

Based on the results from the current study, both bovine milk MUC1 and MUCX are present in skim milk whey or MFGM, which are main by-products of the cheese industry. To find practical uses of these mucins will be economically significant to the dairy industry. Human milk mucins have been reported to prevent adherence of S-fimbriated Escherichia coli to buccal epithelial cells (Schroten et al., 1993). Moreover, high molecular weight mucin-like components from bovine milk have inhibitory activity for Helicobacter pylori, a human gastric pathogen causing chronic gastritis and duodenal ulcer disease (Hirmo et al., 1998). Also, it has been shown that, in human milk, many components that can protect the young from infections by microorganisms are glycoconjugates (Hamosh, 1998; Peterson et al., 1998). Bovine milk MUC1 and MUCX are high in carbohydrates that are believed to have important functions in this regard. In this sense, MUCX, seemingly carrying more complex carbohydrate structures than MUC1, may have potential to bind a wider spectrum of pathogenic microorganisms and prevent more infectious diseases.

\section{ACKNOWLEDGMENTS}

This research was supported by the Alfred Laboratory of Cheese Chemistry and Technology, South Dakota State University, Brookings. The assistance of the staff at the South Dakota State University Dairy Research and Training Unit in collecting milk samples was greatly appreciated.

\section{REFERENCES}

Akiba, Y., P. H. Guth, E. Engel, I. Nastaskin, and D. Kaunitz. 2000. Dynamic regulation of mucus gel thickness in rat duodenum. Am. J. Physiol. Gastrointest. Liver Physiol. 279:G437-G447.

Baruch, A., M. Hartmann, M. Yoeli, Y. Adereth, S. Greenstein, Y. Stadler, Y. Skornik, J. Zaretsky, N. I. Smorodinsky, I. Keydar, and D. H. Wreschner. 1999. The breast cancer-associated MUC1 gene generates both a receptor and its cognate binding protein. Cancer Res. 59:1552-1561.

Campana, W. M., R. V. Josephson, and S. Patton. 1992. Presence and genetic polymorphism of an epithelial mucin in milk of the goat (Capra hircus). Comp. Biochem. Physiol B 103:261-266.

Escande, F., L. Lemaitre, N. Moniaux, S. K. Batra, J. P. Aubert, and M. P. Buisine. 2002. Genomic organization of MUC4 mucin gene. Towards the characterization of splice variants. Eur. J. Biochem. 269:3637-3644.

Hamosh, M. 1998. Protective function of proteins and lipids in human milk. Biol. Neonate 74:163-176.

Hirmo, S., S. Kelm, M. Iwersen, K. Hotta, Y. Goso, K. Ishihara, T. Suguri, M. Morita, T. Wadstrom, and R. Schauer. 1998. Inhibition of Helicobacter pylori sialic acid-specific haemagglutination by human gastrointestinal mucins and milk glycoproteins. FEMS Immunol. Med. Microbiol. 20:275-281.

Huott, M. L., R. V. Josephson, J. R. Hens, G. W. Rogers, and S. Patton. 1995. Polymorphic forms of the epithelial mucin, PAS-I (MUC1), in milk of Holstein cows (Bos taurus). Comp. Biochem. Physiol. B Biochem. Mol. Biol. 111:559-565.

Johnson, V. G., D. E. Greenwalt, P. J. Madara, and I. H. Mather. 1988. Purification and characterization of a differentiation-specific sialoglycoprotein of lactating-guinea-pig mammary tissue. Biochem. J. 251:507-514.

Komatsu, M., M. E. Arango, and K. L. Carraway. 2002. Synthesis and secretion of Muc4/sialomucin complex: Implication of intracellular proteolysis. Biochem. J. 368(Pt 1):41-48.

Laemmli, U. K. 1970. Cleavage of structural proteins during the assembly of the head of bacteriophage T4. Nature 227:680-685.

Ligtenberg, M. J., L. Kruijshaar, F. Buijs, M. van Meijer, S. V. Litvinov, and J. Hilkens. 1992. Cell-associated episialin is a complex containing two proteins derived from a common precursor. J. Biol. Chem. 267:6171-6177.

Mather, I. H. 2000. A review and proposed nomenclature for major proteins of the milk-fat globule membrane. J. Dairy Sci. 83:203-247.

McManus, J. F. A. 1946. Histologic demonstration of mucin after periodic acid. Nature 158:202.

Morrissey, J. H. 1981. Silver stain for proteins in polyacrylamide gels: a modified procedure with enhanced uniform sensitivity. Anal. Biochem. 117:307-310.

Neville, D. M., Jr., and H. Glossmann. 1974. Molecular weight determination of membrane protein and glycoprotein subunits by dis- 
continuous gel electrophoresis in dodecyl sulfate. Methods Enzymol. 32(Part B):92-102.

Pallesen, L. T., M. H. Andersen, R. L. Nielsen, L. Berglund, T. E. Petersen, L. K. Rasmussen, and J. T. Rasmussen. 2001. Purification of MUC1 from bovine milk-fat globules and characterization of a corresponding full-length cDNA clone. J. Dairy Sci. 84:2591-2598.

Pallesen, L. T., L. Berglund, L. K. Rasmussen, T. E. Petersen, and J. T. Rasmussen. 2002. Isolation and characterization of MUC15, a novel cell membrane-associated mucin. Eur. J. Biochem. 269:2755-2763.

Patton, S. 1982. Release of remnant plasma membrane from milk fat globules by Triton X-100. Biochim. Biophys. Acta 688:727-734.

Patton, S. 2001. MUC1 and MUC-X, epithelial mucins of breast and milk. Adv. Exp. Med. Biol. 501:35-45.

Patton, S., S. J. Gendler, and A. P. Spicer, 1995. The epithelial mucin, MUC1, of milk, mammary gland and other tissues. Biochim. Biophys. Acta 1241:407-424.

Patton, S., and G. E. Huston. 1986. A method for isolation of milk fat globules. Lipids 21:170-174.

Patton, S., G. E. Huston, R. Jenness, and Y. Vaucher. 1989. Differences between individuals in high-molecular weight glycoproteins from mammary epithelia of several species. Biochim. Biophys. Acta 980:333-338.

Patton, S., and L. D. Muller. 1992. Genetic polymorphism of the epithelial mucin, PAS-I, in milk samples from the major dairy breeds. J. Dairy Sci. 75:863-867.

Patton, S., and R. S. Patton. 1990. Genetic polymorphism of PAS-I, the mucin-like glycoprotein of bovine milk fat globule membrane. J. Dairy Sci. 73:3567-3574.
Pearse, A. G. E. 1980. Histochemistry. Theoretical and Applied. Vol. 2. Churchill Livingstone, London, UK.

Peterson, G. L. 1983. Determination of total protein. Methods Enzymol. 91:95-119.

Peterson, J. A., S. Patton, and M. Hamosh. 1998. Glycoproteins of the human milk fat globule in the protection of the breast-fed infant against infections. Biol. Neonate 74:143-162.

Rossi, E. A., R. R. McNeer, S. A. Price-Schiavi, J. M. H. Van den Brande, M. Komatsu, J. F. Thompson, C. A. C. Carraway, N. L. Fregien, and K. L. Carraway. 1996. Sialomucin complex, a heterodimeric glycoprotein complex. Expression as a soluble, secretable form in lactating mammary gland and colon. J. Biol. Chem. 271:33476-33485.

Schroten, H., R. Plogmann, F. G. Hanisch, J. Hacker, R. Nobis-Bosch, and V. Wahn. 1993. Inhibition of adhesion of S-fimbriated E. coli to buccal epithelial cells by human skim milk is predominantly mediated by mucins and depends on the period of lactation. Acta Paediatr. 82:6-11.

Shimizu, M., and K. Yamauchi. 1982. Isolation and characterization of mucin-like glycoprotein in human milk fat globule membrane. J. Biochem. (Tokyo) 91:515-524.

Shimizu, M., K. Yamauchi, Y. Miyauchi, T. Sakurai, K. Tokugawa, and R. A. McIlhinney. 1986. High-Mr glycoprotein profiles in human milk serum and fat-globule membrane. Biochem. J. 233:725-730.

Snow, L. D., D. G. Colton, and K. L. Carraway. 1977. Purification and properties of the major sialoglycoprotein of the milk fat globule membrane. Arch. Biochem. Biophys. 179:690-696.

Towbin, H., T. Staehelin, and J. Gordon. 1979. Electrophoretic transfer of proteins from polyacrylamide gels to nitrocellulose sheets: procedure and some applications. Proc. Natl. Acad. Sci. $76: 4350-4354$. 